\title{
Parameters Optimization of Low Carbon Low Alloy Steel
}

\section{Annealing Process}

Maoyu ZHAO ${ }^{1,2) \dagger}$ and Qianwang $C H E N^{1)}$

1) School of Chemistry and Materials Science, University of Science and Technology of China, Hefei 230026, China

2) Department of Mechanical Engineering, Hefei University, Hefei 230601, China

[Manuscript received 18 August 2012, in revised form 3 December 2012]

(C) The Chinese Society for Metals and Springer-Verlag Berlin Heidelberg

\begin{abstract}
A suitable match of annealing process parameters is critical for obtaining the fine microstructure of material. Low carbon low alloy steel $(20 \mathrm{CrMnTi})$ was heated for various durations near Ac temperature to obtain fine pearlite and ferrite grains. Annealing temperature and time were used as independent variables, and material property data were acquired by orthogonal experiment design under intercritical process followed by subcritical annealing process (IPSAP). The weights of plasticity (hardness, yield strength, section shrinkage and elongation) of annealed material were calculated by analytic hierarchy process, and then the process parameters were optimized by the grey theory system. The results observed by SEM images show that microstructure of optimization annealing material are consisted of smaller lamellar pearlites (ferrite-cementite) and refining ferrites which distribute uniformly. Morphologies on tension fracture surface of optimized annealing material indicate that the numbers of dimple fracture show more finer toughness obviously comparing with other annealing materials. Moreover, the yield strength value of optimization annealing material decreases apparently by tensile test. Thus, the new optimized strategy is accurate and feasible.
\end{abstract}

KEY WORDS: Annealing; Process parameters; Pearlite; Grey system; Optimization

\section{Introduction}

Cold forging is one of the modern advanced forming techniques widely used in manufacturing mechanical parts. Usually, low carbon low alloy steel has to be annealed before cold forging to obtain microstructure with larger elongation, section shrinkage, lower yield strength and hardness. The pearlite of the initial material is usually coarse, and the annealing process can improve the plasticity of the steel by changing its microstructure into lamellar pearlites.

Heating temperature and holding time are important process parameters for obtaining fine microstructure. Several articles have recommended that the steels should be heated into the temperature region between the upper and lower critical temperatures

\footnotetext{
† Corresponding author. Prof., Ph.D.; Tel: +86 55162159313 ; Fax: +86 55162159313 ; E-mail address: chhmyzhao@126.com (Maoyu ZHAO)
}

DOI: $10.1007 / \mathrm{s} 40195-012-0141-1$ prior to spheroidizing the carbides below the lower critical temperature. Annealing of medium carbon steel (AISI 4140) under A1 temperature holding for various times was investigated by Karadeniz ${ }^{[1]}$, who found that the highest performance can be reached within $12 \mathrm{~h}$ spheroidization time. In addition, it has been demonstrated that spheroidizing time has the most influence $(58.5 \%)$ on spheroidized percentage and that the initial microstructure only has $31.1 \%$ contribution $^{[2]}$. Hernandez-Silva et al. ${ }^{[3]}$ demonstrated that the degree of spheroidization was accelerated significantly by heat treatments consisting of intercritical process followed by subcritical annealing. Moreover, they found that the subcritical process requires much less time for spheroidization than the intercritical process for medium carbon steels ${ }^{[4]}$.

Several studies on spheroidizing annealing process and its mechanism for medium carbon steels have been conducted. The spheroidized microstructures, with finely distributed spherical cementite particles in a ferrite matrix, have the advantage of enhancing the 
already excellent ductility in medium carbon steels. The introduced mechanisms, such as Rayleigh's capillarity induced perturbation theory ${ }^{[5-7]}$, grain boundary thermal groove theory ${ }^{[8,9]}$, are useful in describing the progressive disruption of the lamellar cementite well. However, the roles of process optimization on spheroidizing are less understood, and the relationship between process parameters optimization and plasticity of low carbon low alloy steel has been rarely investigated.

The purpose of this article is to investigate the influence of annealing parameters on properties of material by IPSAP. Desired properties, such as lower hardness and yield strength as well as better elongation and section shrinkage, were obtained through optimizing annealing parameters.

\section{Rational Grade of Grey System}

The grey system theory was proposed in 1982 by a well-known Chinese scientist, Professor Juilong Deng. Since 1982, the grey system theory as a new theory and method has been widely applied in the fields of sociology, economy, science and technology in China and in other parts of the world.

Grey relational analysis is a problem-solving method when dealing with similarity measures of complex relations in the grey system theory. The bigger the value of grey rational grade is, the better the similarity of factor is. Optimal process parameters can be determined through the grey relational grade as the performance index ${ }^{[10]}$. The method of grey relative analysis can be used to analyze the relation between two grey factors.

We call the series $X$ and $X_{i}$, which are the same measure of a comparable series. $X=\{x(k)\}, X_{i}=$ $\left\{x_{i}(k)\right\}$, where $i=1,2, \cdots, m$ is the gather factor, $k=1,2, \cdots, n$ is the gather index. The reference sequence is given as $X_{0}=\left\{x_{0}(k)\right\}$, the given sequence might be $X_{i}=\left\{x_{i}(k)\right\}$. The definition of the grey relational grade in the grey relational analysis is to show the relational degree between the sequences of $X_{0}(k)$ and $X_{i}(k)$, the grey relational generation can be calculated as

$\xi_{i}(k)=\frac{\min _{i} \min _{k}\left|x_{0}(k)-x_{i}(k)\right|+\rho \max _{i} \max _{k}\left|x_{0}(k)-x_{i}(k)\right|}{\left|x_{0}(k)-x_{i}(k)\right|+\rho \max _{i} \max _{k}\left|x_{0}(k)-x_{i}(k)\right|}$

where $\rho$ is the resolution coefficient which is generally in $[0.0,0.5]$, the value of $\rho$ is 0.5 in this study. $\left|x_{0}(k)-x_{i}(k)\right|$ denotes the absolute value of the difference between $x_{0}(k)$ and $x_{i}(k)$, $\min _{i} \min _{k}\left|x_{0}(k)-x_{i}(k)\right|$ is the smallest value of $\min _{k}\left|x_{0}(k)-x_{i}(k)\right|$ and $\max _{i} \max \left|x_{0}(k)-x_{i}(k)\right|$ is the largest value of $\max _{k}\left|x_{0}{ }^{i}(k)-x_{i}(k)\right|$.

The grey rational grade $\gamma_{i}$ is called the related degree of $X_{0}(k)$ and $X_{i}(k)$. Then, $\gamma_{i}$ is defined as

$$
\gamma_{i}=\frac{1}{n} \sum_{k=1}^{n} \lambda_{k} \xi_{i}(k)
$$

where $\lambda_{k}$ is the weight of the gather object.

The investigation for annealing parameter optimization can be considered a problem of grey systems. This research includes known information or the influence of annealing parameters of effecting on material properties, and also unknown information or how the influence of annealing parameters effecting on the properties, so the mechanisms are adapted to the process optimization.

\section{Experimental Procedure}

\subsection{Schemes of experiment process}

Annealing is a complicated process of microstructure evolution. Process parameters, such as heating temperature and holding time, are important for obtaining fine microstructure.

Cold-rolled 20CrMnTi steel was used as the initial material in this study. The chemical composition of the steel employed in this study is shown in Table 1. Hardness $\rho_{\mathrm{v}}$, elongation $\delta$, section shrinkage $\psi$, and yield strength $\sigma_{\mathrm{s}}$ of the raw material are $140 \mathrm{HV}, 33 \%, 69 \%$ and $495 \mathrm{MPa}$ respectively. Process parameters which are heating temperature $T_{1}$ (holding time $\left.t_{1}\right)$ of intercritical process, heating temperature $T_{2}$ (holding time $t_{2}$ ) of subcritical process, were taken as independent variables. The orthogonal experiment was established with the plastic object of $\rho_{\mathrm{v}}, \delta, \psi$, and $\sigma_{\mathrm{s}}$, as well as 3 level and 4 factors $\left(L_{9}\left(3^{4}\right)=9\right)$, these parameters are designed and listed in Table 2 . The tensile specimen with a reduced gauge length of $35 \mathrm{~mm}$ and a $5 \mathrm{~mm}$ diameter gauge section is depicted in Fig. 1.

\subsection{Experimental method}

Lamellar cementites in hypoeutectoid steel could be dissolved into smaller lamellar easily below the critical temperature $A_{\mathrm{c}}$. The cementites in mild carbon steel ( 0.2 wt.\% C) can be totally dissolved in lamellar pearlites (ferrite-cementite) below $A_{c}$ in the annealing process. ${ }^{[11,12]}$ In this study, heating temperature of intercritical process were set at $T_{1}\left(T_{1} \geq A_{\mathrm{c} 1}\right)$ and held in order to obtain lamellar cementites during intercritical process. The reason is that lamellar cementites are dissolved entirely and coarse cementites may be suppressed to reduce during followed subcritical process. Then, heating temperature of subcritical process were reduced from $T_{1}$ to $T_{2}\left(A_{\mathrm{r} 1}<T_{2}<A_{\mathrm{c} 1}\right)$, and once again held at $T_{2}$ enough to refine lamellar

Table 1 Chemical composition of 20CrMnTi steel (wt.\%)

\begin{tabular}{cccccccccc}
\hline $\mathrm{C}$ & $\mathrm{Si}$ & $\mathrm{Mn}$ & $\mathrm{P}$ & $\mathrm{S}$ & $\mathrm{Ti}$ & $\mathrm{Cr}$ & $\mathrm{Cu}$ & $\mathrm{Ni}$ & $\mathrm{Fe}$ \\
\hline 0.16 & 0.26 & 1.0 & 0.025 & 0.030 & 0.06 & 1.1 & 0.18 & 0.15 & Bal. \\
\hline
\end{tabular}


Table 2 Process parameters and experiment result

\begin{tabular}{|c|c|c|c|c|c|c|c|c|}
\hline \multirow[t]{2}{*}{ Experimental No. } & \multicolumn{4}{|c|}{ Process parameter } & \multicolumn{4}{|c|}{ Result } \\
\hline & $T_{1}\left({ }^{\circ} \mathrm{C}\right)$ & $T_{2}\left({ }^{\circ} \mathrm{C}\right)$ & $t_{1}(\mathrm{~h})$ & $t_{2}(\mathrm{~h})$ & $\rho_{\mathrm{v}}(\mathrm{HV})$ & $\sigma_{\mathrm{s}}(\mathrm{MPa})$ & $\delta(\%)$ & $\psi(\%)$ \\
\hline 1 & 740 & 700 & 5 & 4 & 102.6 & 468.6 & 36 & 76.5 \\
\hline 2 & 740 & 710 & 6 & 5 & 103.3 & 463.5 & 40 & 77.9 \\
\hline 3 & 740 & 720 & 7 & 6 & 101.4 & 449.8 & 42 & 78.6 \\
\hline 4 & 750 & 700 & 6 & 6 & 125.2 & 451.6 & 31 & 72.6 \\
\hline 5 & 750 & 710 & 7 & 4 & 122.3 & 420.2 & 30 & 72.9 \\
\hline 6 & 750 & 720 & 5 & 5 & 118.3 & 408.5 & 36 & 76.0 \\
\hline 7 & 760 & 700 & 7 & 5 & 112.4 & 401.4 & 32 & 74.3 \\
\hline 8 & 760 & 710 & 5 & 6 & 126.5 & 414.7 & 28 & 71.8 \\
\hline 9 & 760 & 720 & 6 & 4 & 120.1 & 414.3 & 36 & 76.5 \\
\hline
\end{tabular}

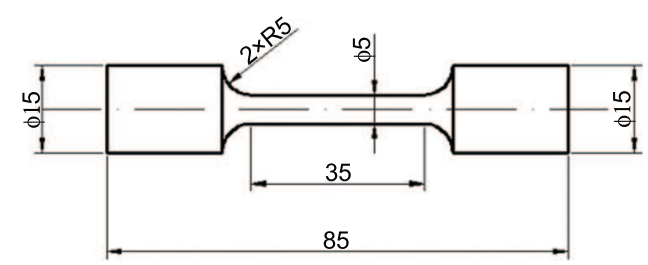

Fig. 1 Geometry and dimensions in millimeters for tensile tests (unit: $\mathrm{mm}$ )

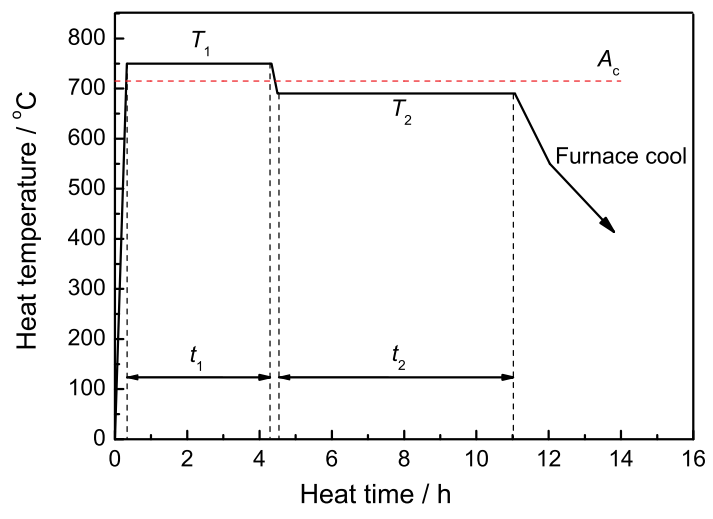

Fig. 2 Annealing history

cementites. Temperatures of $A_{\mathrm{c} 1}$ and $A_{\mathrm{r} 1}$ for the sample $(20 \mathrm{CrMnTi})$ are $740{ }^{\circ} \mathrm{C}$ and $680{ }^{\circ} \mathrm{C}$ respectively. And then, the annealing temperature was dropped to $500{ }^{\circ} \mathrm{C}$ in a furnace of vacuum, and the specimens were finally furnace-cooled to room temperature. The annealing parameter design is given in Table 2. Fig. 2 shows the process parameters of $T_{2}, t_{2}, T_{1}, t_{1}$, and annealing history.

\section{Results}

$\delta, \psi$, and $\sigma_{\mathrm{s}}$ of all annealing materials were measured through tensile tests, and $\rho_{\mathrm{v}}$ was obtained by Vicker's hardness test. The results are listed in Table 2 .

\subsection{Object weight AHP calculation}

Analytic hierarchical process (AHP) that can deal with complex decision was used in the present work. A satisfactory strategy can be attained through the alternative comparison method introduced by Saaty et $a l^{[13]}$. When the factor weights have been found using fuzzy analytic hierarchy process, factors that cause more reasonable behavior in the system could be determined ${ }^{[14]}$. A set of criteria and sub-criteria elements was evaluated through a series of pair comparisons, as reported by Garcia-Cascales et al. ${ }^{[15]}$, to solve a specific class of problems that involve prioritization of potential alternate solutions.

AHP was used to obtain the weight of each criterion. The scale of importance in Saaty's AHP is defined as follows ${ }^{[16]}$ : 1 for equal importance; 3 for moderate importance; 5 for strong importance; 7 for very strong or demonstrated importance; 9 for extreme importance; and 2, 4, 6, 8 for compromise.

In the AHP, the degree of preference of the decision maker in the choice of each pairwise comparison is quantified on a scale of $1-9$, and the quantities are placed in a matrix of comparisons. Even numbers (2, 4,6 , and 8) can be used to represent to compromises among the preferences below. Fig. 3 shows the hierarchy of the problem. The first level of the hierarchy shows that the general goal is to select the best plasticity. At the second level, four criteria $\left(t_{2}, T_{2}, t_{1}, T_{1}\right)$ will contribute to the achievement of the overall goal. Finally, at the third level, each plasticity alternative $\left(\rho_{\mathrm{v}}, \psi, \delta, \sigma_{\mathrm{s}}\right)$ contributes to each criterion in a unique way.

Readers are referred to Saaty ${ }^{[16]}$ for obtaining comparison matrix of the criteria in Table 3. An expert in the end determined the preference numbers in the choice for each pairwise comparison. In Table 3, the comparison matrix of the criteria and the priority weights are shown. Saaty's consistency ratio, consistency index/random index, was used to test the consistency of the eigenvector of the comparison matrix of the criteria. The consistency ratio of the matrix in Table 3 was obtained as 0.092 . Since this ratio is less than 0.10 , the comparison matrix is reasonable consistent.

The pairwise comparison matrices of evaluating the material plasticity with respect to process parameters are given in the following Tables 4-7. Then, calculate eigenvector corresponding to eigenvalue of maximum from the pairwise comparison matrices with AHP theory.

The weight vector from Table 4 is calculated as $W_{t 2}=(0.1407,0.2583,0.6370,0)$. The weight value of 


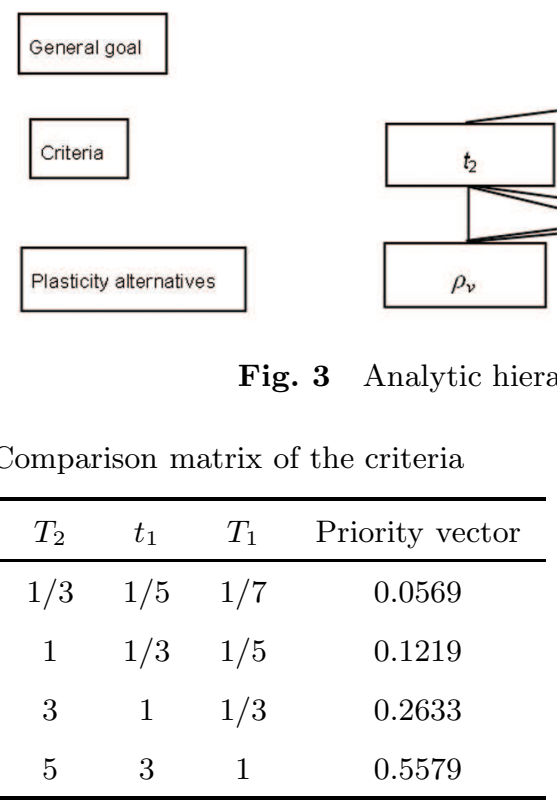

Table 4 Evaluation of the material plasticity with respect to $t_{2}$

\begin{tabular}{ccccc}
\hline Alternative & $\rho_{\mathrm{v}}$ & $\psi$ & $\delta$ & $\sigma_{\mathrm{s}}$ \\
\hline$\rho_{\mathrm{v}}$ & 1 & $1 / 3$ & $1 / 5$ & 0 \\
$\psi$ & 3 & 1 & $1 / 3$ & 0 \\
$\delta$ & 5 & 3 & 1 & 0 \\
$\sigma_{\mathrm{s}}$ & 0 & 0 & 0 & 0 \\
\hline
\end{tabular}

Table 5 Evaluation of the material plasticity with respect to $T_{2}$

\begin{tabular}{ccccc}
\hline Alternative & $\rho_{\mathrm{v}}$ & $\psi$ & $\delta$ & $\sigma_{\mathrm{s}}$ \\
\hline$\rho_{\mathrm{v}}$ & 0 & 0 & 0 & 0 \\
$\psi$ & 0 & 1 & $1 / 2$ & $1 / 3$ \\
$\delta$ & 0 & 2 & 1 & $1 / 2$ \\
$\sigma_{\mathrm{s}}$ & 0 & 3 & 2 & 1 \\
\hline
\end{tabular}

Table 6 Evaluation of the material plasticity with respect to $t_{1}$

\begin{tabular}{ccccc}
\hline Alternative & $\rho_{\mathrm{v}}$ & $\psi$ & $\delta$ & $\sigma_{\mathrm{s}}$ \\
\hline$\rho_{\mathrm{v}}$ & 1 & 7 & 0 & 5 \\
$\psi$ & $1 / 7$ & 1 & 0 & $1 / 3$ \\
$\delta$ & 0 & 0 & 0 & 0 \\
$\sigma_{\mathrm{s}}$ & $1 / 5$ & 3 & 0 & 1 \\
\hline
\end{tabular}

$t_{2}$ relative to $\delta$ is 0.6370 which shows the influence of $t_{2}$ effecting on $\delta$ is the maximum. The other matrices of pairwise comparisons and the weight vector of each matrix are given in the following.

The weight vector from Table 5 is calculated as $W_{T 2}=(0,0.1634,0.2969,0.5396)$. The weight value of $T_{2}$ relative to $\sigma_{\mathrm{s}}$ is 0.5396 which shows the influence of $T_{2}$ effecting on $\sigma_{\mathrm{s}}$ is the maximum.

The weight vector from Table 6 is calculated as $W_{t 1}=(0.7306,0.0810,0,0.1884)$. The weight value of
Table 7 Evaluation of the material plasticity with respect to $T_{1}$

\begin{tabular}{ccccc}
\hline Alternative & $\rho_{\mathrm{v}}$ & $\psi$ & $\delta$ & $\sigma_{\mathrm{s}}$ \\
\hline$\rho_{\mathrm{v}}$ & 1 & 0 & 5 & 3 \\
$\psi$ & 0 & 0 & 0 & 0 \\
$\delta$ & $1 / 5$ & 0 & 1 & $1 / 2$ \\
$\sigma_{\mathrm{s}}$ & $1 / 3$ & 0 & 2 & 1 \\
\hline
\end{tabular}

Table 8 Results of AHP for priority weight of plasticity

\begin{tabular}{cccccc}
\hline & $t_{2}$ & $T_{2}$ & $t_{1}$ & $T_{1}$ & Priority weight \\
\hline Weight & 0.0569 & 0.1219 & 0.2633 & 0.5579 & \\
$\rho_{\mathrm{v}}$ & 0.1407 & 0 & 0.7306 & 0.6483 & 0.5621 \\
$\sigma_{\mathrm{s}}$ & 0.2583 & 0.1634 & 0.0810 & 0 & 0.2435 \\
$\delta$ & 0.6370 & 0.2969 & 0 & 0.1220 & 0.1405 \\
$\psi$ & 0 & 0.5396 & 0.1884 & 0.2297 & 0.0559 \\
\hline
\end{tabular}

$t_{1}$ relative to $\rho_{\mathrm{v}}$ is 0.7306 which shows the influence of $t_{1}$ effecting on $\rho_{\mathrm{v}}$ is the maximum.

The weight vector from Table 7 is calculated as $W_{T 1}=(0.6483,0,0.1220,0.2297)$. The weight value of $T_{1}$ relative to $\rho_{\mathrm{v}}$ is 0.6483 which shows the influence of $T_{1}$ effecting on $\rho_{\mathrm{v}}$ is the maximum.

Table 8 shows the combination of the weight vectors obtained by pairwise comparisons. From Table 8 , the consistency ratio is 0.019 which is also below 0.10 , the comparison matrix is reasonable consistent. As a result, the reasonable weights of $\rho_{\mathrm{v}}, \sigma_{\mathrm{s}}, \delta$, and $\psi$ are $\lambda_{1}=0.5621, \lambda_{2}=0.2435, \lambda_{3}=0.1405$ and $\lambda_{4}=0.0559$, respectively.

\subsection{Grey relational generating calculation}

The ideal reference sequence is $X_{0}=$ $\left\{x_{0}(k)\right\}=(101.4,401.4,42,78.6)$ from Table 2 , the given sequence of plasticity is $X_{i}=\left\{x_{i}(k)\right\}$, where $i=1,2, \cdots, 9$ correspond to experimental No., $k=1,2$, 3,4 . $x_{i}(1), x_{i}(2), x_{i}(3)$, and $x_{i}(4)$ correspond to the values of $\rho_{\mathrm{v}}, \sigma_{\mathrm{s}}, \delta$ and $\psi$ respectively in each experiment from Table 2. The grey relational generations of the material plasticity were calculated by using Eq. (1). The result is listed in Table 9 , where $\xi_{1}, \xi_{2}, \xi_{3}$, and $\xi_{4}$ are the grey relational generation of $\rho_{\mathrm{v}}, \sigma_{\mathrm{s}}, \delta$ and $\psi$, respectively.

\subsection{Grey rational grade calculation}

By incorporating $\lambda_{k}$ and $\xi_{i}(k)$ into Eq. (2), the grey rational grades were calculated and listed in Table 10 . 
With the increase of the grey rational grade $\gamma_{i}$, the material plasticity approaches gradually the optimum value. The values of process parameter corresponding to the maximum values of $\gamma_{\mathrm{i}}$ are $T_{1}=740{ }^{\circ} \mathrm{C}, t_{1}=$ $7 \mathrm{~h}, T_{2}=720{ }^{\circ} \mathrm{C}$ and $t_{2}=5 \mathrm{~h}$, which are the optimum parameters. The average grey rational grade of the parameter is listed in Table 11. The values of $\rho_{\mathrm{v}}$, $\sigma_{\mathrm{s}}, \delta$, and $\psi$ of the material properties annealed by optimum parameters are $110.5 \mathrm{HV}, 328 \mathrm{MPa}, 42.5 \%$ and $82 \%$, respectively. As result, the plasticity of the optimum material with lower hardness and yield strength, as well as better elongation and section shrinkage, has been obviously enhanced.

\section{Discussion}

\subsection{Microstructure}

The starting microstructure of the material is consisted of coarse pearlites and ferrites. Pearlites are white crystal phase, ferrites are black crystal phase respectively as shown in Fig. 4. Results in Figs. 5(a) to $5(\mathrm{i})$ reveal that pearlites of annealing material have non-uniform distribution. However, microstructure of material annealed by optimum parameters in Fig. 5(j) presents that grain sizes of ferrite and pearlite decrease obviously, and show more uniform distribution than others. The grain sizes of ferrite under different annealing are shown in Table 12. Fig. 5 exhibits that pearlites in optimization annealing material are much more homogeneous than other pearlites in unoptimization annealing materials.

Pearlite microstructures of the material are displayed in Fig. 6 under different annealing process. Every interlamellar spacing of cementites was measured by SEM, and then the average value was calculated as shown in Table 13. Table 13 shows that the spacings in Figs. 6(a) to 6(i) are mostly in the range of $208 \mathrm{~nm}$ to $371 \mathrm{~nm}$, it is $227 \mathrm{~nm}$ in the initial material, whereas it is $208 \mathrm{~nm}$ in the optimization annealing material. It was observed that there are more crystal phase interfaces between cementite and ferrite in

Table 9 Grey relational generation of plasticity

\begin{tabular}{ccccc}
\hline Experiment No. & $\xi_{1}$ & $\xi_{2}$ & $\xi_{3}$ & $\xi_{4}$ \\
\hline 1 & 0.9831 & 0.5102 & 0.5385 & 0.7692 \\
2 & 0.9736 & 0.5299 & 0.7778 & 0.9091 \\
3 & 1 & 0.5912 & 1 & 1 \\
4 & 0.7463 & 0.5824 & 0.3889 & 0.5385 \\
5 & 0.7701 & 0.7883 & 0.3684 & 0.5512 \\
6 & 0.8055 & 0.9079 & 0.5385 & 0.7292 \\
7 & 0.8642 & 1 & 0.8750 & 0.6195 \\
8 & 0.7361 & 0.8403 & 0.3333 & 0.5072 \\
9 & 0.7892 & 0.8444 & 0.5385 & 0.7692 \\
\hline
\end{tabular}

Table 10 Grey rational grade of plasticity in different experiments

\begin{tabular}{ccccccccc}
\hline No.1 & No.2 & No.3 & No.4 & No.5 & No.6 & No.7 & No.8 & No.9 \\
\hline 0.1989 & 0.2091 & 0.2256 & 0.1615 & 0.1768 & 0.1976 & 0.2217 & 0.1734 & 0.1920 \\
\hline
\end{tabular}

Table 11 Average grey rational grade of the process parameter

\begin{tabular}{cccccccc}
\hline$T_{1}\left({ }^{\circ} \mathrm{C}\right)$ & $\gamma_{\mathrm{i}}$ & $T_{2}\left({ }^{\circ} \mathrm{C}\right)$ & $\gamma_{\mathrm{i}}$ & $t_{1}(\mathrm{~h})$ & $\gamma_{\mathrm{i}}$ & $t_{2}(\mathrm{~h})$ & $\gamma_{\mathrm{i}}$ \\
\hline 740 & 0.2112 & 700 & 0.1940 & 7 & 0.2080 & 6 & 0.1868 \\
750 & 0.1786 & 710 & 0.1864 & 6 & 0.1875 & 5 & 0.2095 \\
760 & 0.1957 & 720 & 0.2051 & 5 & 0.1900 & 4 & 0.1892 \\
\hline
\end{tabular}
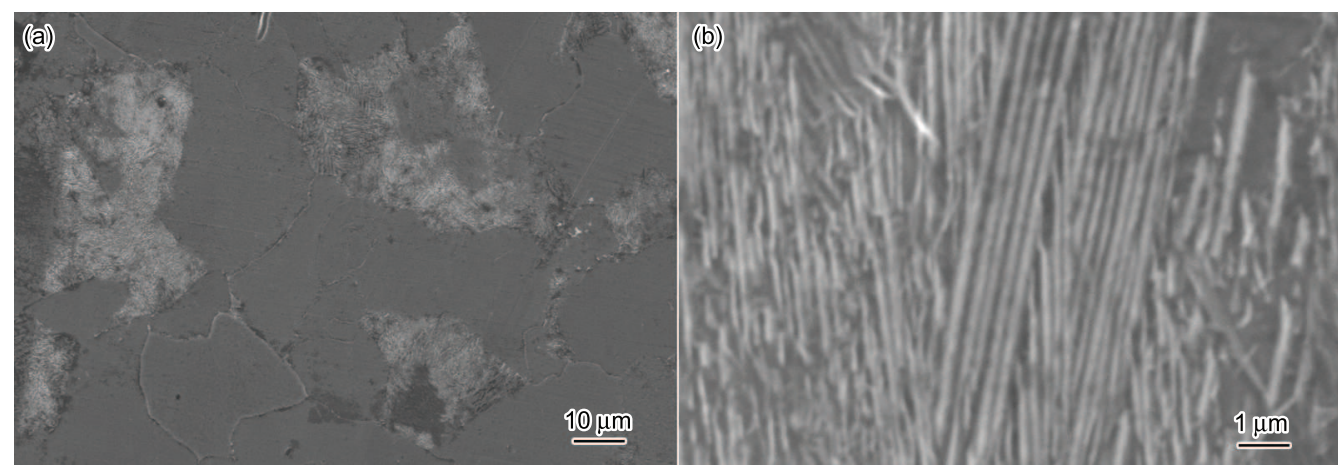

Fig. 4 SEM images of initial 20CrMnTi steel 


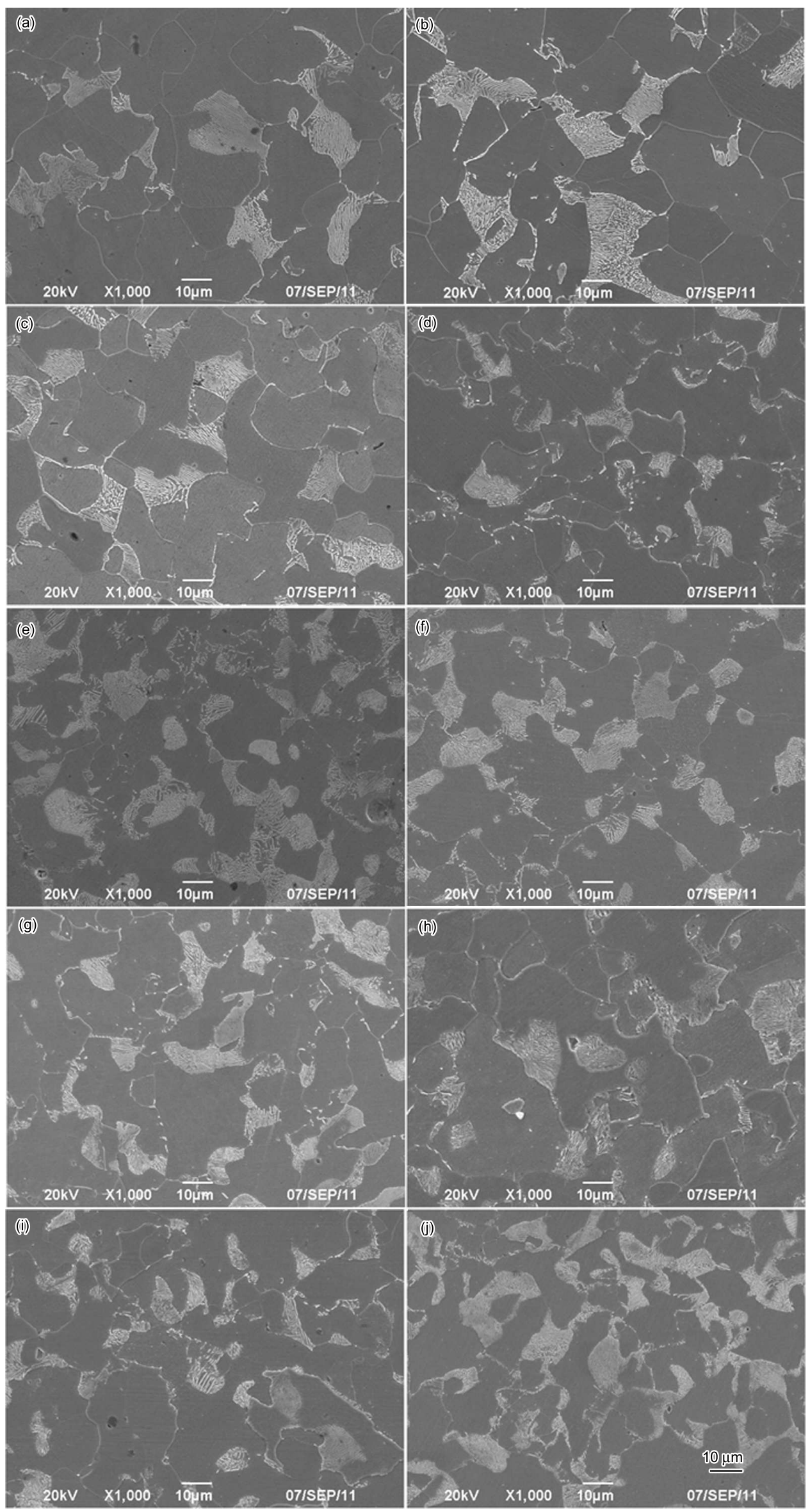

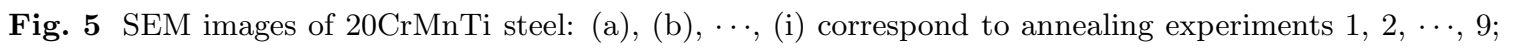
(j) optimization annealing experiment 
Table 12 Grain sizes $(\mu \mathrm{m})$ of ferrite in different experiments and the opimization

\begin{tabular}{cccccccccc}
\hline No.1 & No.2 & No.3 & No.4 & No.5 & No.6 & No.7 & No.8 & No.9 & Optimization \\
\hline 21.7 & 17.1 & 18.8 & 15.2 & 14.0 & 17.8 & 15.7 & 18.7 & 16.5 & 16.7 \\
\hline
\end{tabular}
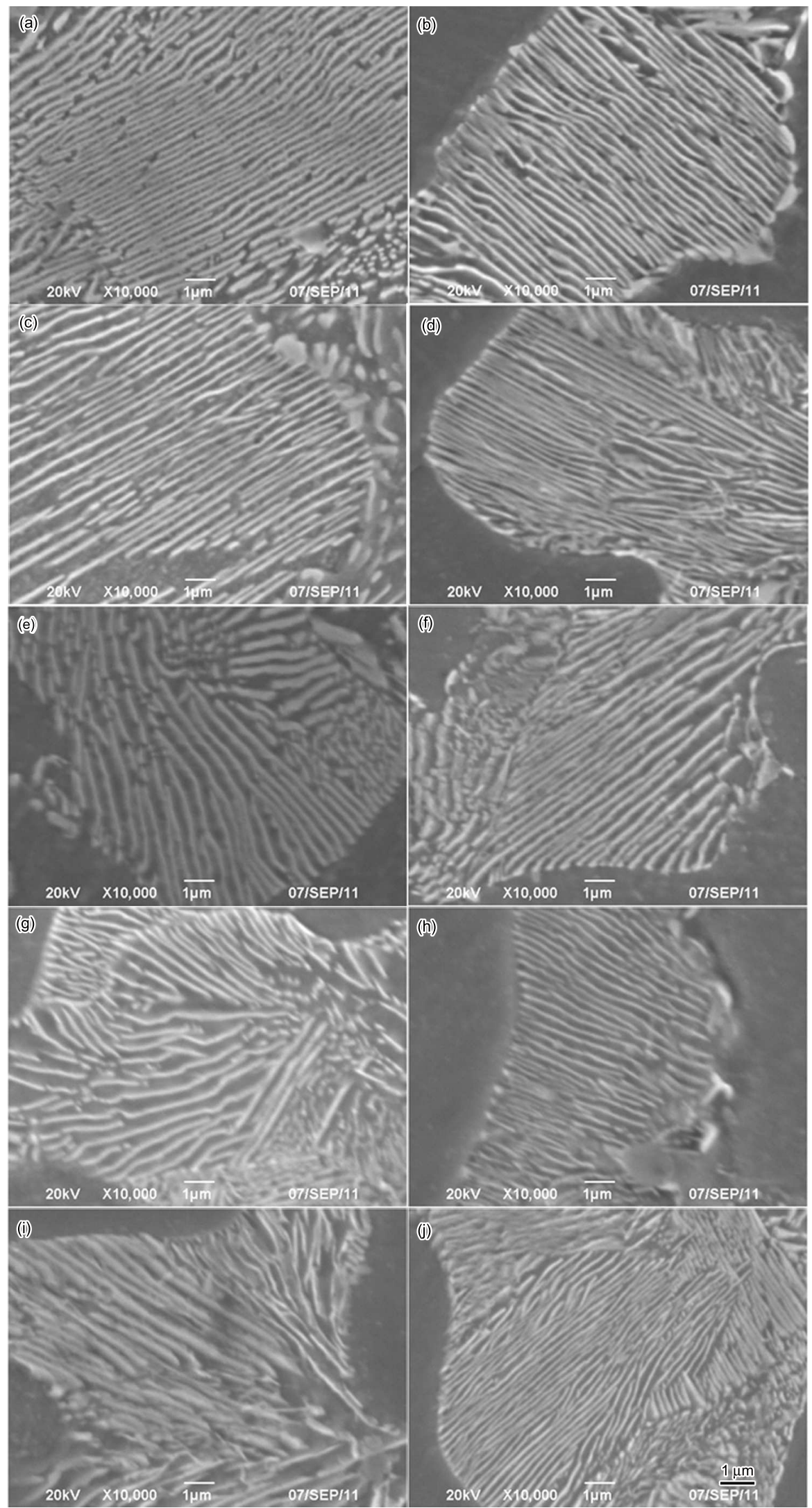

Fig. 6 SEM images showing the pearlite microstructures of $20 \mathrm{CrMnTi}$ steel: (a), (b), , , (i) correspond to annealing experiments $1,2, \cdots, 9$; (j) optimization annealing experiment 
Table 13 Interlamellar spacings $(\mathrm{nm})$ of cementite in different experiments and the opimization

\begin{tabular}{cccccccccc}
\hline No.1 & No.2 & No.3 & No.4 & No.5 & No.6 & No.7 & No.8 & No.9 & Optimization \\
\hline 217 & 255 & 243 & 208 & 275 & 255 & 301 & 284 & 371 & 184 \\
\hline
\end{tabular}

Fig. 6(j), and the interlamellar spacing of cementites in optimization annealing material is much smaller and the smaller lamellar cementites are also evident in Fig. 6(j). The plasticity could be strengthened by increasing the contact area between cementite and ferrite. Therefore, the smaller lamellar cementites are of benefit to plasticity because it is easier for sliding, deforming and bending by loading.

The interlamellar spacing of cementite is depended on annealing temperature and time. When the transformation of austenitizing occurs during intercritical process, the austenitizing rate is controlled primarily by carbon diffusion. Austenitizing had happened at $740{ }^{\circ} \mathrm{C}\left(A_{\mathrm{c} 1}\right)$ and cementites began to dissolve. Then the temperature was down to $720{ }^{\circ} \mathrm{C}$ (below $A_{\mathrm{c} 1}$ ) and held for some time, carbon can absorb more energy leading to diffusing sufficiently. When annealing temperature was down below $A_{\mathrm{r} 1}$, cementites began to form and grew slowly. Above results suggest that the dissolving scale of lamellar cementites was bigger than the growth scale of pearlite during the optimization process. It is considered that this behavior might result in the formation of smaller ferrite-pearlite grains distributed uniformly.

\subsection{X-ray fluorescence spectrometer tests}

Chemical compositions of original sample and annealing samples were measured by X-ray fluorescence spectrometer. Element contents of $\mathrm{C}$ and $\mathrm{S}$ are 0.155 wt.\% and 0.029 wt.\% in original sample, 0.154 wt.\% and $0.034 \mathrm{wt} . \%$ in the sample of experimental No. 3 and $0.154 \mathrm{wt} . \%$ and $0.034 \mathrm{wt} . \%$ in the sample of optimization annealing experiment. The data shown in Table 14 prove that the contents of $\mathrm{C}$ and $\mathrm{S}$ in samples had no missing almost during annealing. The results demonstrate that the material plasticity of optimization annealing was promoted greatly due to smaller interlamellar cementites.

\subsection{Fracture surfaces and tensile tests}

Ferrite-pearlite grains which are uniformly distributed make plasticity deformation perfectly, smaller lamellar cementites can reduce resistance of dislocation motion and increase formability by promoting deformation slip, and it is easier for cold forging compared with coarse cementites. SEM images of

Table 14 Chemical composition of sample (wt.\%)

\begin{tabular}{cccc}
\hline $\begin{array}{c}\text { Element } \\
\text { content }\end{array}$ & Original & $\begin{array}{c}\text { Experiment } \\
\text { No.3 }\end{array}$ & $\begin{array}{c}\text { Optimization } \\
\text { experiment }\end{array}$ \\
\hline C & 0.155 & 0.154 & 0.154 \\
S & 0.029 & 0.034 & 0.034 \\
\hline
\end{tabular}

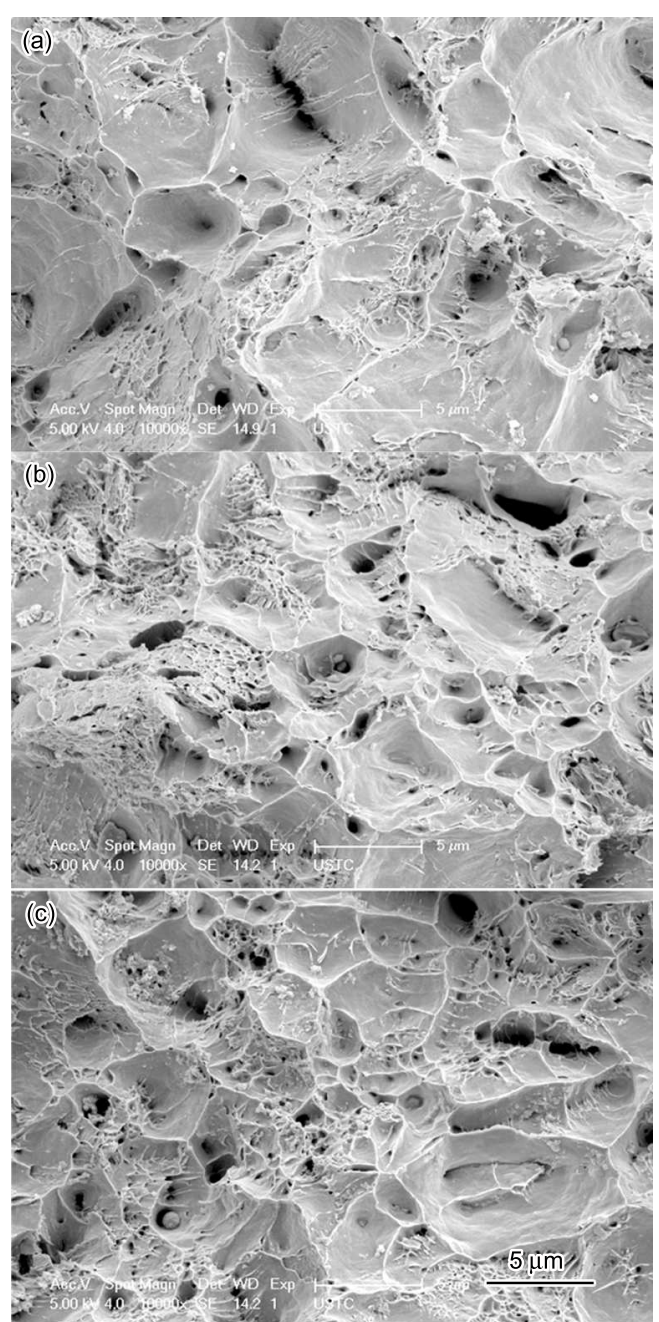

Fig. 7 SEM images of fracture surfaces after tensile tests: (a) initial; (b) experiment No. 3; (c) optimization annealing experiment

fracture surfaces after tensile tests in Fig. 7 show clearly the morphology of the tension fracture surface. The numbers of dimples are much more, and its width-depth is bigger in the optimization annealing material than other annealing materials. The results are in accordance with above microstructure characterization and this implies that the optimization annealing material has better plasticity.

On comparsion of the tensile stress vs. strain curves in Fig. 8, the curve of the material annealed by optimum parameters indicates an evident decrease in deformation forces, and the yield strength is also reduced dramatically measured by the tensile test. Fig. 8 and Table 2 show that the yield strength is in the range of $328-495 \mathrm{MPa}$, and is $495 \mathrm{MPa}$ for initial material, whereas $328 \mathrm{MPa}$ for the optimization annealing material. This might be caused by the uniform distribution of ferrites and smaller lamellar cementites inside pearlites during optimization 


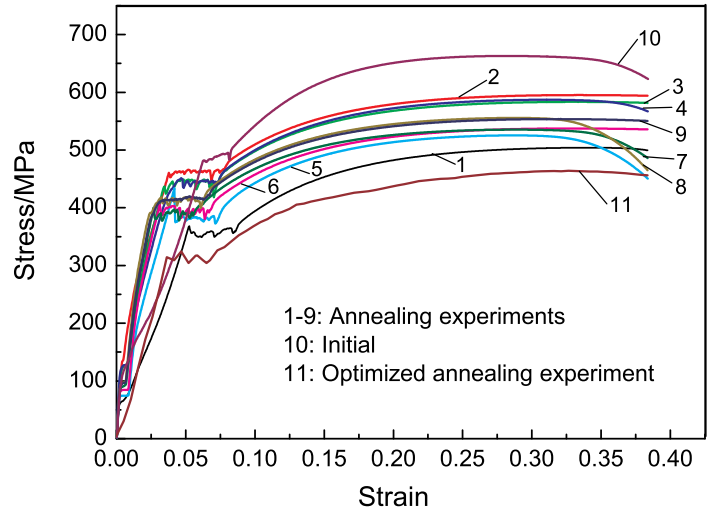

Fig. 8 Comparison of tensile stress vs. strain curves of the samples in different experiments and the opimization

annealing. Though smaller grains can improve material strength, the uniform distribution of lamellar pearlites may be plays a more important role in decreasing material strength. Therefore, there could be considerable improvements in the plasticity of low carbon low alloy steel (20CrMnTi) with optimized annealing parameters.

\section{Conclusions}

(1) The weights of annealing parameters, such as intercritical heating temperature $T_{1}$ and holding time $t_{1}$, subcritical heating temperature $T_{2}$ and holding time $t_{2}$, were calculated using AHP theory, and they are $0.5621,0.2435,0.1405$ and 0.0559 , respectively.

(2) The optimized annealing parameters $T_{1}, t_{1}, T_{2}$, and $t_{2}$ of $20 \mathrm{CrMnTi}$ material by IPSAP are $740{ }^{\circ} \mathrm{C}$, $7 \mathrm{~h}, 720{ }^{\circ} \mathrm{C}$ and $5 \mathrm{~h}$, respectively.

(3) The results observed through microstructure confirm that the interlamellar spacings of cementites and grain sizes of ferrites in the optimized annealing material are smaller. In addition, the distribution of ferrites and pearlites in the material is much more uniform than others.
(4) The yield strength of the optimized annealing material is reduced dramatically, and there are much more and bigger dimples on fracture surfaces after tensile tests. The result also shows clearly that the plasticity of the optimized annealing material is obviously promoted.

\section{Acknowledgements}

The authors are grateful to the Talent Science Foundation of Hefei University (No. 10RC02) and the Anhui Province Colleges and Universities Science Foundation (No. KJ2011ZD09) for the financial support.

\section{REFERENCES}

[1] E. Karadeniz, Mater. Des. 29 (2008) 251.

[2] A. Kamyabi-Gol and M. Sheikh-Amiri, Iron. Steel. Res. Int. 17 (2010) 45.

[3] D. Hernandezsilva, R.D. Morales and J.G. Cabanasmoreno, ISIJ Int. 32 (1992) 1297.

[4] J.M. O'brien and W.F. Hosford, Metall. Mater. Trans. A 33 (2002) 1255.

[5] Y.L. Tian and R.W. Kraft, Metall. Mater. Trans. A 18 (1987) 1359.

[6] E. Werner, Acta Metall. 37 (1989) 2047.

[7] S.A. Hackney, Scr. Metall. 25 (1991) 799.

[8] W.W. Mullins, Trans. AIME 218 (1960) 354.

[9] Y.G. Nakagawa and G.C. Weatherly, Met. Trans. A 3 (1972) 3223.

[10] H.V. Trivedi and J.K. Singh, Biosystems Eng. 92 (2005) 521.

[11] M.J. Harrigan and O.D. Sherby, Mater. Sci. Eng. 7 (1971) 177

[12] J. Agren, H. Abe, T. Suzuki and Y. Sakuma, Metall. Mater. Trans. A 17 (1986) 617.

[13] T.L. Saaty and N. Begicevic, Appl. Soft Comput. 10 (2010) 963.

[14] M. Dagdeviren and I. Yueksel, Inf. Sci. 178 (2008) 1717.

[15] M.S. Garcia-Cascales and M.T. Lamata, Comput. Ind. Eng. 56 (2009) 1442.

[16] T.L. Saaty and H.S. Shih, Euro. J. Oper. Res. 199 (2009) 867. 